\title{
Réécriture : ingestions, greffes et remakes poétiques espagnols actuels
}

\section{David Gondar}

\section{(2) OpenEdition}

1 Journals

Édition électronique

URL : https://journals.openedition.org/cher/2315

DOI : 10.4000/cher.2315

ISSN : 2803-5992

Éditeur

Presses universitaires de Strasbourg

\section{Édition imprimée}

Date de publication : 29 mai 2018

Pagination : 121-131

ISBN : 979-10-344-0019-5

ISSN : 1968-035X

\section{Référence électronique}

David Gondar, «Réécriture : ingestions, greffes et remakes poétiques espagnols actuels », reCHERches [En ligne], 20 | 2018, mis en ligne le 01 décembre 2021, consulté le 02 décembre 2021. URL : http:// journals.openedition.org/cher/2315; DOI : https://doi.org/10.4000/cher.2315

\section{(c) (i) (2)(2)}

Ce(tte) œuvre est mise à disposition selon les termes de la Licence Creative Commons Attribution Pas d'Utilisation Commerciale - Partage dans les Mêmes Conditions 4.0 International. 


\title{
Réécriture : ingestions, greffes et remakes poétiques espagnols actuels
}

\author{
DAVID GONDAR ${ }^{1}$
}

Un texte peut toujours en lire un autre, et ainsi de suite jusqu'à la fin des textes.

Gérard Genette, Palimpsestes.

$\ll \int \begin{aligned} & \text { e est un hôte } »^{2} \text { affirme lécrivain et critique Julien Frantz, proposant une } \\ & \text { réécriture du pôle d'identité rimbaldien comme un intrus ou un parasite. }\end{aligned}$ Le poète et le poème sont alors contaminés par cette intrusion. La réécriture est un procédé qui par l'imitation ou la recréation propose un éclairage nouveau d'un texte modifiant ses intentions et la réception du public. Des brouillons aux reprises comme la cinquantaine de prologues de Museo de la Novela de la Eterna (1975) de Macedonio Fernández ou «Pierre Menard, autor del Quijote» (1944) de Jorge Luis Borges, transforment le texte de départ, un hypotexte selon la terminologie de Genette. Lorsque Pierre Ménard réécrit la première partie de Don Quijote (1605), il introduit des références culturelles postérieures à Miguel de Cervantès, comme l'influence des philosophes Friedrich Nietzsche, Bertrand Russell et William James. L'imitation se limite dans ce cas à une réécriture littérale car elle se voit augmentée de références culturelles contemporaines à Borges. Les innombrables prologues de Macedonio Fernández sont proches d'une réécriture sans fin car comme il le signale dans sa «Quadruple préface?» (151):

la Littérature est précisément le belart de: exécuter artistiquement un sujet découvert par d'autres. C'est la loi de tout belart et cela signifie que le «sujet» d'art n'a pas de valeur artistique: l'exécution fait toute la valeur de l'art.

1 Docteur en littérature espagnole contemporaine et enseignant en Langue et littérature espagnoles à l'Université de Strasbourg et en école d'ingénieurs. Thèse: «Les voies de la poésie espagnole actuelle (1990-2010): marginalités, hybridations, porosité, intermédialité », dirigée par Isabelle Reck, soutenue le 8 décembre 2016.

2 Vers du poème «Syzygie» construit en diptyque avec le poème «Sol Niger» (1999). 
De la sorte, Borges employait la surcharge pour annoter ses propres livres ou ses lectures. La relation à son œuvre se créait à partir du texte imprimé et les annotations de lecture établissaient une cartographie et un catalogue. La publication étant alors une nécessité pour la réécriture. La surcharge complète également l'hypotexte comme dans le recueil Poemas lisiados (2012) de Jorge Riechmann où l'écriture manuscrite complète les poèmes typographiés. Cette action apporte une intimité et un ajout sémantique. La surcharge comme élément biffé introduit une modification de l'écriture par l'auteur ou le personnage. Par exemple, La maison des feuilles (2000) de Mark Z. Danielewski se construit à partir de la double lecture - essai sur un film et annotations en bas de page rendant compte d'une multitude de registres, se jouant à la fois de références élitistes et d'un langage familier. La typographie se compose d'éléments barrés, de couleurs et de structures complexes qui réécrivent l'hypotexte. L'hypertexte est alors un produit de jeux typographiques éclairant le lecteur sur la maison, le concept central de l'œuvre. Ce même jeu de surcharge est fortement présent dans Albert Angelo (1964) de B.S. Johnson, L'extravagant voyage du jeune et prodigieux T.S. Spivet (2009) de Reif Larsen et O Révolutions (2006), à nouveau de Mark Z. Danielewski. Ces annotations structurées en tabulation complètent le texte principal, l'éclairent et renseignent le lecteur.

La transcription de récits oraux est un éternel recommencement, pour preuve l'Iliade et l'Odyssée que Virgile reprend dans l'Énéide. Tel le palimpseste des copistes du Moyen-Âge, la réécriture s'articule sur des textes antérieurs. Jean Mambrino explique cette relation à l'écriture, ce dialogue incessant, dans la quatrième de couverture de son recueil de poésie Le Palimpseste (1991):

Mais n'y a-t-il pas sous le premier texte un autre texte plus ancien, dont le dernier n'est que le souvenir, ou le pressentiment? Et qui parle en ces dialogues traduits du silence, ouverts sur la cime, la caverne, la mer? Est-ce le Moi et la Conscience? Dante et Béatrice? L'Âme et l'Esprit? L'inconnu et l'Innomé? Leur échange infiniment singulier, au sein de l'universel désir, rejoint l'intimité de tous ceux qui élaborent en secret, peut-être même à leur insu, la métamorphose du temps en éternité.

La réécriture n'est pas à concevoir comme une simple redite du texte premier mais comme une actualisation qui permet d'insérer des anachronismes et des variations. Il n'y a pas trahison de l'œuvre mais un apport de la singularité de l'hypertexte à celle déjà présente dans l'hypotexte. La translation littéraire est une perception propre au traducteur de l'œuvre qu'il traduit. Lors d'une conférence, le traducteur Claro signalait qu'il n'était pas un passeur mais une "passoire " ${ }^{3}$. La translation d'une langue à une autre modifie le texte en réduisant ou en élargissant le sens. Ce renouvellement proposé par la réécriture cherche à atteindre un nouveau public comme le signalait Roland Barthes dans Le plaisir du texte (1993: 58-59):

3 Bernard Hoepffner et Christophe Claro, Traduire l'Europe, Librairie Kléber, salle blanche, Strasbourg, 10 mars 2009. 
Lisant un texte rapporté par Stendhal (mais qui n'est pas de lui) j'y retrouve Proust par un détail minuscule [...] je comprends que l'œuvre de Proust est, du moins pour moi, l'œuvre de référence, la mathésis générale, le mandala de toute la cosmogonie littéraire [...]. Et c'est bien cela l'intertexte: l'impossibilité de vivre hors du texte infini.

La recherche d'innovations sémantiques et formelles de la poésie espagnole actuelle la rapproche de nouvelles disciplines et techniques, principalement chez Agustín Fernández Mallo, Vicente Luis Mora et Alejandro Céspedes. Elle se propose de faire dialoguer le savoir et la littérature à travers l'intertextualité, l'hypertextualité et l'hybridation, notions se rattachant à la réécriture. Il peut s'agir d'un jeu translittéraire déplaçant le roman vers l'essai, la science vers la poésie, la publicité vers la littérature. C'est ce que font les "postpoètes» ${ }^{4}$ de la «Génération Mutante». Les choix esthétiques dans le nouveau tournant du siècle sont marqués par des partis pris forts qui restent cependant connectés aux œuvres antérieures, même si Agustín Fernández Mallo et Manuel Vilas le nient parfois ironiquement, car c'est dans les avancées technologiques et les nouveaux modes de communication que la plupart des "postpoètes" se reconnaissent avant tout, en cherchant à se laisser contaminer par tout ce qui les entoure. Si la "postpoésie» affiche son rejet du passé, c'est pour mieux l'incorporer. Voilà son paradoxe. Les créations contemporaines, dans leur porosité, ont absorbé des formes venant d'autres genres littéraires ou non. La poésie devient poreuse et s'imprègne de la littérature mais aussi de tout ce qui compose notre société, de la publicité à l'architecture, des sciences aux séries télévisées. Elle propose le plus souvent, d'abord, une réflexion sur l'objet-poème, le lecteur-spectateur et l'espace de la poésie. L’intermédialité s'est développée avec Internet, la vidéo, la musique mais aussi selon les formats de la publicité et de la bande dessinée. Hybride, la poésie se fait caméléon jusqu'à s'imprégner des univers les plus étrangers à son domaine comme les sciences, créant des zones de contact productives, riches autant pour l'une que pour les autres. L'intertextualité est dans l'excès, excès des citations qui confond l'œuvre avec ses références comme dans Construcción (2005) de Vicente Luis Mora. Dans ce recueil, les citations constituent l'intégralité du texte proposé en ouverture, "Primera piedra». La citation n'est pas adhésion au passé mais une manière d'ajouter du monde au monde comme le rappelle Vicente Luis Mora: «se añade mundo al mundo ${ }^{5}$. Le poète contemporain s'approprie, en les "pillant», les œuvres antérieures, mais recycle aussi toute sorte de matériaux non-littéraires. La citation chez les poètes est ainsi de l'ordre du recyclage: par exemple, récupérer des éléments d'un film

4 La "poésie postpoésie» (poesía postpoesía) émerge dans les années 2000 avec les travaux d'Agustín Fernández Mallo et se développe parallèlement à la "Génération Mutante» (Generación Mutante) avec des auteurs comme: Germán Sierra, Juan Francisco Ferré, Javier Calvo, Jorge Carrión, Eloy Fernández Porta, Robert JuanCantavella ou Javier Moreno.

5 Entretien avec Alejandra Vanesa: http://www.literaturas.com/v010/sec0601/ entrevistas/entrevistas-03.htm. 
pour recréer l'espace d'action d'un poème ou créer une métaphore à partir d'un langage mathématique. Le poète sample la société de sa culture à son identité et fabrique un poème "hors norme», dans le sens qu'il va au-delà de la norme, mais aussi et surtout par son caractère impérialiste: il absorbe tout, il est débordant dans l'excès d' «inter»: intertextualités et intermédialités. Comme l'a signalé Mora, si l'éclairage romantique du monde extérieur l'intéresse, c'est pour mieux exprimer un nihilisme qui aveugle.

La «postpoésie» n'est pas une poésie qui cherche des filiations mais un mouvement mutant qui se transforme au contact d'autres œuvres artistiques, sociales ou scientifiques. Elle est un art du présent qui joue des simultanéités et conjoint tous les temps.

Crear es copiar. La materia de la que está hecho el ser humano es la copia. La esencia del ojo es la copia. La esencia de las células es la copia. La esencia del recién nacido es la copia. La esencia de la Red es la copia. Nos encanta que nos copien. Dios creó el Mundo copiándose a sí mismo (Quimera mars 2009).

Voilà un terme, "copier», utilisé en trompe-l'œil, qui tout en évoquant le très contemporain et usuel "copier-coller", renvoie d'une part à des concepts de l'histoire de la littérature, notamment à la question de nos classiques, de l'imitatio et, d'autre part, à des problématiques socio-politiques et culturelles: la copie dans la société de consommation de masse. La copie est ici un système créatif "postpoétique» permettant l'appropriation, dans des zones de contact, des éléments les plus disparates et opposés. La copie, comme la e-littérature, rend possible l'extension, les ramifications d'une œuvre. Dans Carne de píxel, par exemple, Fernández Mallo insère des passages de l'article scientifique «Los agujeros negros, Constructores del Cosmos" (El País 2 novembre 2005) de Mónica Salomé. Non seulement il les versifie, mais il construit aussi une nouvelle structure et donc un nouveau texte, un autre ordre symbolique et systématique. L'insertion d'extraits d'articles scientifiques dans les vers est complétée par la juxtaposition de références musicales (Costa Brava) ou artistiques (Andy Warhol). La copie élargit le public, les supports et les modes d'émergence, en opérant à travers l'expérimentation et l'esthétique. Dans ce sens, elle tente de toucher, voire de réveiller, un lectorat curieux et cultivé qui saurait jongler avec les références des différents champs culturels et scientifiques et qui y trouverait du plaisir. La poésie et les poètes revisitent les canons traditionnels à la recherche d'un renouvellement esthétique constant. Il faut donc comprendre la "postpoésie» comme un projet littéraire cherchant des zones de contact avec tout ce qui compose nos sociétés. Elle puise dans des éléments populaires (publicités, produits de tous les jours), artistiques (cinéma, musique) ou scientifiques (articles, théorèmes). Elle cherche à être omniprésente. Être en contact avec tous les médias et toutes les formes présentes dans les sociétés contemporaines permet à la poésie d'être ultraconnectée et donc ultraproductive. La nouvelle poésie espagnole souhaite ne plus être cataloguée comme une forme à part, au

6 Autrement dit, il échantillonne en retravaillant différents éléments qu’il a rassemblés. 
sens où son langage se différencie de celui de la communication médiatique ou publicitaire mais l'assimilation et l'appropriation de toutes les formes engendrées par la société risquent de faire perdre à la "postpoésie» son caractère poétique au point que celle-ci ne soit plus perçue comme poésie.

Au chapitre 9 de son essai Postpoesía (2009: 173-182), intitulé "RIZOMA», Fernández Mallo emprunte clairement le concept de rhizome de Deleuze et Guattari. Il le cite tout en insérant des commentaires. Le procédé n'est pas un simple acte de citation, de comparaison ou d'explication, il fait du texte Mille Plateaux (Deleuze/ Guattari 1980) un manifeste "postpoétique».

Si la poésie est objet de transtextualité c'est bien à des fins de métatextualié, nous rappelle Gérard Genette dans Palimpsestes (1982). Fernández Mallo conçoit de la sorte son essai, non comme un manifeste, mais comme une théorie appliquée où la réflexion poétique se fait poésie, ouvrant la voie à un essai poétique. La lecture de Mille Plateaux dans l'essai de Fernández Mallo est un acte poétique transtextuel. L'hypertextualité et la métatextualité s'imbriquent en permanence ${ }^{7}$.

Il ne s'agit pas de se situer dans la continuité d'une œuvre mais de s'en servir pour faire œuvre. Le simulacre est, dans la conception grecque, eidôlon, l'idole. Il s'oppose à l'icône qui est copie ou imitation. Dans le simulacre, nous avons une vérité du réel qui remplace celle de départ. Nous ne sommes pas face à une copie ou un jeu d'imitation mais face à une autre œuvre qui existe sans la présence de la première. Nous sommes dans la simulation et non l'imitation, précise le poète et critique Jorge Fernández Gonzalo: "No se trata de una copia, no se busca la reduplicación, sino establecer parámetros de diferencias, pautas de contraste, desvíos" (Filosofía zombi 2011: 169). L'hypertextualité propose à l'œuvre de prendre un autre chemin qui n'est pas de compléter l'œuvre première mais bien de donner autre chose, une autre facette, un univers parallèle. Pour Fernández Gonzalo, le remake $e^{8}$ est à la fois création artistique et commentaire, au croisement de l'hypertextualité et de la métatextualité ${ }^{2}$ définies par Genette.

La «postpoésie» est dans l'immédiateté, dans une mémoire courte qui rompt avec les formes antérieures. Elle ne doit rien aux mouvements passés, aux poètes

7 Hypertextualité: «J'entends par là toute relation unissant un texte B (que j’appellerai hypertexte) à un texte antérieur A (que j'appellerai, bien sûr, hypotexte) sur lequel il se greffe d'une manière qui n'est pas celle du commentaire.» (Genette, 1982: 11).

8 «[...] un remake siempre interpreta el original, establece canales de correspondencia, lecturas, alteraciones que, en ultimo término, nos dan la clave estructural del texto primero, ponen de relieve sus estilemas principales, acumula, mediante la desviación, juegos de perspectiva y registros de lectura que, en lugar de anular o rescribir el original, lo propulsan, lo encaraman a su esencia, restituyen su experiencia de ser, para dejar fuera [...] los efectos de la exégesis y el poder acaparador de la lectura.» (Jorge Fernández Gonzalo 2011: 171).

9 Métatextualité: « est la relation, on dit plus couramment de "commentaire", qui unit un texte à un autre texte dont il parle, sans nécessairement le citer (le convoquer), voire, à la limite, sans le nommer [...]» (Genette, 1982: 9). 
ou aux institutions. Cependant, elle se sert des formes antérieures et les transpose ailleurs. Elle se veut une littérature du déplacement et non une littérature figée, anachronique, incapable d'adaptation. Fernández Mallo reprend les théories de Deleuze et Guattari sur les oppositions entre mémoire à court terme et à long terme, entre l'herbe et l'arbre de Mille Plateaux. Il n'y a pas dépassement des propositions de ces deux penseurs dans les propositions du poète, mais il cherche à les actualiser dans un contexte contemporain hyperconnecté. Au fil de la lecture, le jeu poreux de Fernández Mallo induit le lecteur en erreur. Le texte de Deleuze et Guattari devient le texte de Fernández Mallo. La disposition en paragraphe fait apparaître les citations plus ou moins longues qui s'enchaînent comme des citations suivies de commentaires. Cependant, quelques repères permettent de comprendre que ce qui s'expose là, c'est la contamination du rhizome par la «postpoésie»: insertions de citations en italiques et numérotation des pages citées. Dans cette construction, les voix s'entremêlent jusqu'à faire émerger un concept nouveau: le rhizome dans la «postpoésie». C'est bien la transformation de la lecture du texte d'origine par la porosité littéraire qui fait de l'hypotexte non plus un texte antérieur mais une écriture en écho. Les textes A et $\mathrm{B}$ se rejoignent dans une annulation du temps. L'enjeu de la "postpoésie» est l'atemporalité par une ouverture totale à tout apport extérieur et dans toute sa diversité. La porosité transforme le texte ou l'élément de départ (formule mathématique, slogan publicitaire, ou un autre poème, un vers, une ligne d'un essai, un essai) qui est ainsi inséré dans un système "postpoétique», et acquiert une valeur "postpoétique». Cette porosité même facilite le chemin inverse: le retour de cet élément dans son espace premier. Comme pour le rhizome, devenu "rhizome postpoétique», il peut à nouveau sortir du champ de la poésie espagnole actuelle, mais il reste rattaché par sa greffe littéraire et conceptuelle à la "postpoésie». La mutation que recherche Fernández Mallo est celle qui permet au poète d'être en perpétuel mouvement.

Dans son adaptation de El hacedor (1960) de Borges, Il reprend l'œuvre originale, c'est-à-dire une œuvre mêlant essais, poèmes et récits. Son objectif est de créer une nouvelle version qui s'agglutine à l'œuvre de départ et se confonde avec elle:

pero mañana yo también habré muerto y se confundirán nuestros tiempos, y la cronología se perderá en un orbe de símbolos premodernos y de algún modo será justo afirmar que yo le he traído este libro y que usted lo ha aceptado (2011: 10).

Le concept d' «appropriationnisme» défendu par la "postpoésie» n'est pas recevable selon María Kodama, la veuve de Jorge Luis Borges, qui fait interdire le remake de Fernández Mallo. Une lettre défendant le droit à la création fut signée par des écrivains, universitaires et étudiants tels que: Jorge Carrión, Juan Villoro, Rosa Montero, Sergio Gaspar, Pablo García Casado, Manuel Vilas, Vicente Luis Mora, Javier Moreno, Luna Miguel, Jorge Riechmann, Jordi 
Doce, etc. ${ }^{10}$. François le Lionnais, membre de l'Oulipo, définit l'appropriation par la temporalité à travers le concept de "plagiat par anticipation ${ }^{11}$ dans La littérature potentielle (1973:21):

Il nous arrive parfois de découvrir qu'une structure que nous avions crue parfaitement inédite, avait déjà été découverte ou inventée dans un passé lointain. Nous nous faisons un devoir de reconnaître cet état de choses en qualifiant les textes en cause de plagiats par anticipation.

Fernández Mallo indique que l' «appropriationnisme» est la «fundición en el presente de objetos poéticos de todas las épocas y tendencias» (Postpoesía: 67). Il va même plus loin, reprenant un entretien d'Andy Warhol, de 1963:

¿Cómo alguien puede decir que algún estilo es mejor que otro? Uno debería ser capaz de ser expresionista abstracto la próxima semana, o artista pop, o realista, sin sentir que ha renunciado a algo (Postpoesía: 67).

La porosité littéraire permet à la poésie de traverser ses limites afin de tisser une toile lui permettant de se développer sous de nouvelles formes et de nouveaux thèmes et ainsi atteindre un nouveau lectorat. La poésie actuelle instaure des méthodes d'exploration permettant aux poètes de puiser dans la symbolique postmoderne, restant ultra-connectés à notre environnement. La porosité génère de nouvelles formes (hybridation et intermédialité), qui opère une véritable mutation du poème: un poème en constante dérive entre sa construction et sa finition. Lorsque Melker Garay publie mcv (2014) chez Norlén \& Slottner, il permet à la nouvelle de Jorge Luis Borges, «La Biblioteca de Babel» (1944), de voir une de ses références imaginaires prendre forme.

Uno, que mi padre vio en un hexágono del circuito quince noventa y cuatro, que constaba de las letras $\mathrm{MCV}$, perversamente repetidas desde el renglón primero hasta el último (89).

L'auteur suédois, né au Chili, en réalisant le livre évoqué par Borges et en déposant le copyright, en devient l'auteur et s'introduit ainsi dans l'œuvre du maître argentin. C'est l'appropriation d'un texte et d'une idée. Garay parvient à récupérer, d'une part, un élément de «La Biblioteca de Babel» mais, d'autre part, il est aussi récupéré par la nouvelle, par ce qu'il nomme «une des prophéties de Borges ${ }^{12}$. Si l'auteur érige un acte littéraire borgésien lorsqu'il publie un livre composé uniquement des lettres « $\mathrm{m}$ », «c» et « V» sur 410 pages, l'éditeur Norlén \& Slottner par contre, par la publication limitée à deux cents exemplaires, nonvendus en librairie, ne situe pas le livre dans la création littéraire mais comme un objet d'art contemporain destiné aux cabinets de curiosités.

10 http://fernandezmallo.megustaleer.com/2011/10/06/carta-por-la-retirada-de-elhacedor-remake/.

11 Déjà au XVIII siècle, le poète et dramaturge Alexis Piron dans La métromanie (1738) écrivit: «Leurs écrits sont des vols qu'ils nous ont faits d'avance.» (Acte III, scène 7).

12 Essai de Merkel Garay sur son œuvre $m c v$ : http://www.garay.se/english/mcv/. 
L’intermédialité de la poésie espagnole contemporaine proposel'appropriation de références allant de la littérature à la publicité, aux sciences ou à l'informatique en passant par la parodie et le plagiat. La frontière entre les signatures s'efface. Par exemple, l'appropriation des mathématiques dans la poésie à des fins métaphoriques repense le nombre tant au niveau du sens que du visuel. Le nombre est un appariement qui met en correspondance des ensembles. Il couple le sens et la graphie, les mathématiques et la poésie. Dans tous les cas, il ne s'agit plus d'emprunter simplement un thème ou un motif aux sciences, comme le signale Javier Moreno, mais de rendre les mathématiques poétiques et la poésie scientifique. Le rapprochement vise bien à une réciproque transformation, à une interaction et à l'émergence de nouvelles manières de lire et de voir le monde qui font interagir métaphore et équation. La réécriture se construit alors comme une recherche systématique et une expansion critique de la littérature et des sciences. Les «métaphores scientifiques» ou «équations poétiques» sont également présentes dans la poésie du poète vénézuélien Daniel Ruiz et des péruviens Enrique Verástegui et Rodolfo Hinostroza. La métatextualité a ici pour objectif une exactitude de la poésie mais également le rapprochement entre des univers éloignés et la construction de nouvelles perspectives communes. Le lien devient alors un moteur d'écriture ou de réécriture, voire de lecture ${ }^{13}$ ou de relecture, permettant à l'œuvre de se défaire d'une structure stable afin de se déformer, de se modifier selon la curiosité et les centres d'intérêt du poète et du lecteur mais aussi du temps qui produit de nouvelles extensions ou les fait évoluer.

La réécriture dans l'œuvre de Ramón Dachs repose à la fois sur la géométrie, la multiplicité d'une vérité et la transcription. Le Taula general du philosophe et poète Llull (1293-1294) fonctionne comme un poème liminaire dans le recueil Codex Mundi (2012). Ce schéma llulien en ouverture, relie l'écriture fractale de Dachs à celle de Llull. La syntaxe linéaire est remplacée par une géométrie qui combine des mots et des concepts pour créer un réseau d'interconnexions et une actualisation des préceptes llulien. La relecture du Taula general est une réécriture augmentée et renouvelée. $L$ '«intersémioticité»n'est plus une forme ou l'autre; elle n'est pas davantage substrat ou superstrat, mais adstrat: les formes ne s'annulent ni ne s'effacent, elles coexistent en un même noyau.

L'une des spécificités de la «postpoésie» est son utilisation de l'ordinateur comme outil majeur de la création, le recours à la publicité, le pastiche, l'emprunt de modèles typologiques d'environnements extérieurs à la poésie, la volonté de s'insérer dans le marché, le divertissement et l'appropriation. L'herméneutique littéraire, par la révolution numérique, devient une nouvelle interprétation des symboles. Comme le signale Nicholas Negroponte dans L'homme numérique (1995) : «l'autoroute numérique va transformer l'art achevé et inaltérable en une chose du passé» (275). La lecture, par son extension informatique et numérique,

13 Dans les années 1970, les théories de la réception et de la lecture de l'École de Constance mettaient en avant la tension existante entre le texte et la lecture, car la lecture est aussi productrice de sens, et donc par cet acte, il y a réécriture du texte. 
est transformée. Le lecteur ne se voit plus seulement restituer la lecture par le texte, mais également par des hyperliens, qui font du «lecteur-spectateur», un acteur. Le texte est en mutation par les modifications apportées par l'auteur, tant dans l'intertextualité que dans l'intermédialité. Fredric Jameson, dans Le postmodernisme ou la logique culturelle du capitalisme tardif (2007), signale que la littérature passe du temps diachronique au synchronique, permettant ainsi d'aller de la modernité à la postmodernité. Le poème est en mouvement par les possibilités offertes par la page Internet. La réception de la poésie se construit autour de la page Internet qui se distingue du livre car elle permet la simultanéité d'éléments (texte, image, son, vidéo) et une lecture «active». Jesús Jiménez Domínguez précise que le poème est interconnecté à d'autres formes comme la vidéo-installation poétique et le poème visuel car l'art et la littérature sont des reflets de la société technologique. Les poètes utilisent les nouvelles technologies comme des instruments pour leurs créations. Il y a donc la possibilité de réécriture avec ces nouveaux mécanismes qui permettent également d'autres avantages et répercussions dans le champ médiatique. Melchor López souligne l'idée de montage dans certains de ses poèmes en prose, devenant des "poèmesscène».

La porosité littéraire de la poésie espagnole du $\mathrm{xxI}^{\mathrm{e}}$ siècle n'est pas uniquement dans la correspondance ou l'hybridation: elle mêle l'intertextualité aux formes hybrides. L'action du lecteur est démultipliée par les nouvelles possibilités numériques. Si les pages du livre se déplacent d'écran en écran, de l'ordinateur au téléphone portable, le poème se transforme par ce changement de contenant. C'est en repensant la transmission du message que le contenu se voit également changé.

Le détournement des messages publicitaires et autres pollutions visuelles comme dans jPod (2006) de Douglas Coupland où le spam des boîtes mails devient un discours dans la narration. Transformer en poésie ce qui ne l'est pas, n'est-ce pas répondre à la mondialisation de la culture qui a généré une esthétique unique dans un système éditorial régi par les lois du marché? La translation du genre "métier de clergie» (Mester de clerecía) dans le recueil de poèmes Mester de Cibervía (2000) de Vicente Luis Mora est une réécriture cherchant un renouvellement à partir d'un système actualisé. Il y a à la fois une variation de la structure, des thèmes et des scènes afin de rendre une signification nouvelle.

En conclusion, la réécriture dans la poésie espagnole actuelle s'efforce de rendre la multiplicité des significations de thèmes et la multiplicité des structures à travers des reprises singulières et novatrices. Lorsque Alejandro Céspedes compose ses poèmes de Flores en la cuneta (2009) à partir de slogans automobiles, il redéfinit la réception du message publicitaire en l'orientant vers l'accident de voiture. Le slogan devenant titre du poème brouille le message premier, la relecture établit alors une nouvelle vérité, une subversion des valeurs initiales. 
L'écrivain (du latin scribanus), est à la fois celui qui écrit et celui qui copie. Il faudra attendre l'époque moderne pour voir naître l'acception spécifique d'auteur d'une œuvre littéraire. L'emprunt littéraire, la greffe, la réécriture, la transcription sont continuité et éclairage nouveau de textes antérieurs. La reprise est un prolongement qui insère une signification contemporaine, des mécanismes et des systèmes inédits. L'adaptation en roman graphique de Nocilla Experience (2008) de Fernández Mallo par Pere Joan, en 2011, n'est autre qu'une nouvelle lecture qui permet la translation d'un genre à un autre (du roman à la bande dessinée) et élargit le public. La relecture est aussi proposée par l'auteur même de l'œuvre comme dans Les Malchanceux (1969) de B.S. Johnson dont le format du livre permet une réflexion et une expérimentation de la mémoire fragmentée.

La «métaphore nouvelle» de la poésie espagnole contemporaine est un noyau poétique qui connaît des embranchements multiples par le rapprochement et l'appropriation de formes et genres habituellement étrangers à la poésie. Si la «postpoésie» est un art de l'appropriation c'est aussi parce que notre société postmoderne est sans cesse dans la réappropriation et la dérivation. La science trouve chez Fernández Mallo et la "postpoésie», dont il est un des poètes les plus paradigmatiques, une nouvelle valeur et une place majeure. Il ne s'agit pas de proposer une poésie scientifique ou une science poétique, mais de donner l'impulsion pour une véritable mutation par l'intégration de tous les éléments qui configurent notre société, la science, avec ses avancées accélérées, étant l'un de ces éléments déterminants dans les évolutions des comportements et du rapport au monde.

L'œuvre est en éternelle gestation, sans cesse complétée et augmentée, ou simplement modifiée. Ainsi, le recueil Cuando llegan las abejas (2012) fusionne les vers de Begoña Callejón et ceux de Sylvia Plath et nous interroge dès le prologue sur l'auteur del'œuvre: "No sé quién soy. Tú o yo» (13).

\section{Bibliographie}

Barthes R., 1993, Le plaisir du texte, Paris, Le Seuil.

Borges J. L., 2001, «Pierre Menard, autor del Quijote», Ficciones, Madrid, Alianza Editorial, coll. «Biblioteca Borges», p. 41-55.

Borges J.L., 2001, «La Biblioteca de Babel», Ficciones, Madrid, Alianza Editorial, p. 86-99.

Borges J.L., 1997, El hacedor, Madrid, Alianza Editorial.

Callejón B., 2012, Cuando llegan las abejas, Cadix, Alumbre, coll. "Poésie», Diputación de Cádiz.

Céspedes A., 2009, Flores en la cuneta, Madrid, Ediciones Hiperión, coll. «Poesía Hiperión».

Coupland D., 2010, jPod, Vauvert, Au Diable Vauvert. 
Dachs R., 2012, Codex Mundi, Madrid, Amargord Ediciones, coll. «pi de poesía » $\mathrm{n}^{\circ} 2$.

Danielewski M.Z., 2007, La maison des feuilles, Paris, Éditions Denoël, coll. «Denoël \& d'Ailleurs».

Danielewski M.Z., 2007, O Révolutions, Paris, Éditions Denoël, «Denoël \& d'Ailleurs".

Deleuze G. et Guattari F., 1980, Capitalisme et Schizophrénie 2. Mille Plateaux, Paris, Éditions de Minuit, coll. "Critique».

Fernández M., 2010, Museo de la Novela de la Eterna, Madrid, Cátedra, coll. «Letras Hispánicas».

Fernández Gonzalo J., 2011, Filosofía zombi, Barcelone, Anagrama, coll. «Argumentos».

Fernández Mallo A., 2008, Nocilla Experience, Madrid, Alfaguara.

Fernández Mallo A., 2011, El hacedor (de Borges), Remake, Madrid, Alfaguara.

Garay M., 2014, mcv, Kristinehamn, Norlén \& Slottner.

Genette G., 1982, Palimpsestes, Paris, Le Seuil.

Jameson F., 2007, Le postmodernisme ou la logique culturelle du capitalisme tardif, Paris, ENSBA.

Johnson B.S., 2009, Albert Angelo, Meudon, Quidam Éditeur, coll. «Made in Europe».

Johnson B.S., 1999, Les malchanceux, Meudon, Quidam Éditeur, coll. «Made in Europe».

Larsen R., 2009, L'extravagant voyage du jeune et prodigieux T.S. Spivet, Paris, Nil Éditions.

Lionnais F. le, 1973, Oulipo. La littérature potentielle, Paris, Gallimard.

Mambrino J., 1991, Le Palimpseste ou les dialogues du désir, Paris, José Corti.

Mora V.L., 2000, Mester de Cibervía, Valence, Pre-textos.

Mora V.L., 2005, Construcción, Valence, Pre-textos.

Negroponte N., 1995, L’homme numérique, Paris, Robert Laffont.

Riechmann J., 2012, Poemas lisiados 2009-2011, Madrid, La Oveja Roja.

Warhol A., 1993, Mi filosofía de A a B y de B a A, Barcelone, Tusquets Editores. 